\title{
Spectroscopy of $\mathrm{Mn}$ atoms isolated in solid ${ }^{4} \mathrm{He}$
}

\author{
P. Moroshkin, ${ }^{\text {a),b) }}$ V. Lebedev, ${ }^{\text {b) }}$ and A. Weis ${ }^{\text {b) }}$ \\ Department of Physics, University of Fribourg, Chemin du Musée 3, 1700 Fribourg, Switzerland
}

\begin{abstract}
We present an experimental study of the laser-induced luminescence spectra of Mn atoms in solid helium matrices. We observe transitions of the valence electron and of inner-shell electrons. We find that the Mn-He interaction perturbs the inner-shell transitions to a lesser extent than the valence-electron transitions. The observed lineshapes of the inner-shell transitions of $\mathrm{Mn}$ are similar to those of an inner-shell transition in Ba studied earlier. At the same time, they are more strongly perturbed than the corresponding transitions in $\mathrm{Au}$ and $\mathrm{Cu}$ under the same conditions. We suggest a qualitative explanation of these observations based on the atomic bubble model. Our results also suggest that the inner-shell transitions of $\mathrm{Mn}$ in solid $\mathrm{He}$ are more strongly perturbed than the same lines of $\mathrm{Mn}$ isolated in solid $\mathrm{Ar}$ or $\mathrm{Kr}$ matrices.
\end{abstract}

\section{INTRODUCTION}

Metal atoms embedded in liquid and solid helium matrices form nanometer-sized cavities, so-called atomic bubbles. The expansion or shrinking of the bubble following an electronic transition of an atom in a bubble produces wavepackets of matrix phonons. The characteristic features of these wavepackets can be derived from the embedded atoms' excitation and fluorescence spectra using methods of laser spectroscopy. ${ }^{1}$ In this context, transitions of inner-shell electrons in transition-metal atoms are particularly interesting since they lead to the excitation of phonon pairs and the observed lineshapes give a direct measure of the phonon energy. Transitions between the inner $4 f$ and $5 d$ shells of the lanthanides Tm and Eu have been studied earlier. ${ }^{2,3}$ In our recent experiments, ${ }^{1,4-6}$ we have addressed transitions between the filled inner $(n-1) d$-shell and the outer $n s$-shell of $\mathrm{Cu}(n=4)$ and $\mathrm{Au}(n=6)$ atoms, as well as a $5 d 6 s-6 s^{2}$ transition of Ba. It was found that the spectral lines of gold and copper follow the same trend as the inner-shell transitions of the lanthanides with pronounced zero-phonon lines (ZPL) and phonon wing (PW) structures. Similar structures have also been observed in the spectra of various molecules isolated in superfluid $\mathrm{He}$ nanodroplets (for a review see Ref. 7). Surprisingly, no ZPL was observed in the spectrum of $\mathrm{Ba}$. At the same time, the $5 d 6 s-6 s^{2}$ transition lineshape in Ba clearly differs from those of the well-studied transitions in the outer shell.

In the present work, we extend those studies to Mn atoms with special attention given to transitions between the partially filled inner $3 d$-shell and the outer $4 s$-shell. We will refer to transitions of this type as "inter-shell" transitions.

The transitions reported here are represented on the Mn term diagram in Fig. 1 with the free-atomic transition wavelengths taken from Ref. 8. The inter-shell transitions under

\footnotetext{
a) Present address: Low Temperature Physics Laboratory, RIKEN, 2-1 Hirosawa, Wako, Saitama 351-0198, Japan. Electronic mail: petr.moroshkin@ riken.jp

b) URL: www.unifr.ch/physics/frap/
}

discussion are forbidden in the free atom. In matrix-isolation studies, however, these lines are readily observed and may even dominate the luminescence spectrum.

It is also interesting to compare the spectra of the same dopant isolated in different matrices. It is expected that difference of the crystalline lattice stiffness and the next-neighbor distances in solid He compared to other rare-gases should lead to different trapping-site structures and produce different perturbations in the luminescence and absorption spectra. Excitation and luminescence spectra of manganese atoms in the heavier solid rare-gas matrices $\mathrm{Ar}, \mathrm{Kr}$, and $\mathrm{Xe}$ were recently investigated in detail. ${ }^{9-12}$

\section{EXPERIMENTS}

\section{A. Experimental setup}

A detailed description of our experimental setup can be found in Refs. 6 and 13. Most measurements were performed in the hep phase of solid ${ }^{4} \mathrm{He}$ at $1.5 \mathrm{~K}$ and $30-33$ bar. The matrix-isolated $\mathrm{Mn}$ sample was produced in a helium pressure cell immersed in superfluid helium and cooled by pumping on the helium bath. The cell was connected to an external high-pressure reservoir that allowed us to grow He crystals by condensing and then pressurizing helium gas.

The solid helium matrix was doped with Mn atoms by means of laser ablation using second harmonic radiation $(\lambda=532 \mathrm{~nm})$ of a pulsed Nd:YAG-laser focused from above onto a solid manganese target at the bottom of the pressure cell.

The implanted $\mathrm{Mn}$ atoms are excited either by the third harmonic of the same pulsed Nd:YAG laser $(\lambda=355 \mathrm{~nm})$ or by a tunable optical parametric oscillator (OPO) pumped by a (another) frequency-tripled pulsed Nd:YAG laser. Luminescence light from the sample volume is collimated by a lens located in the cryostat and is then analyzed by a grating spectrograph ( $0.1 \mathrm{~nm}$ resolution) equipped with a CCD camera. With this spectrometer and the camera, we could cover a spectral range of 350-950 $\mathrm{nm}$. 


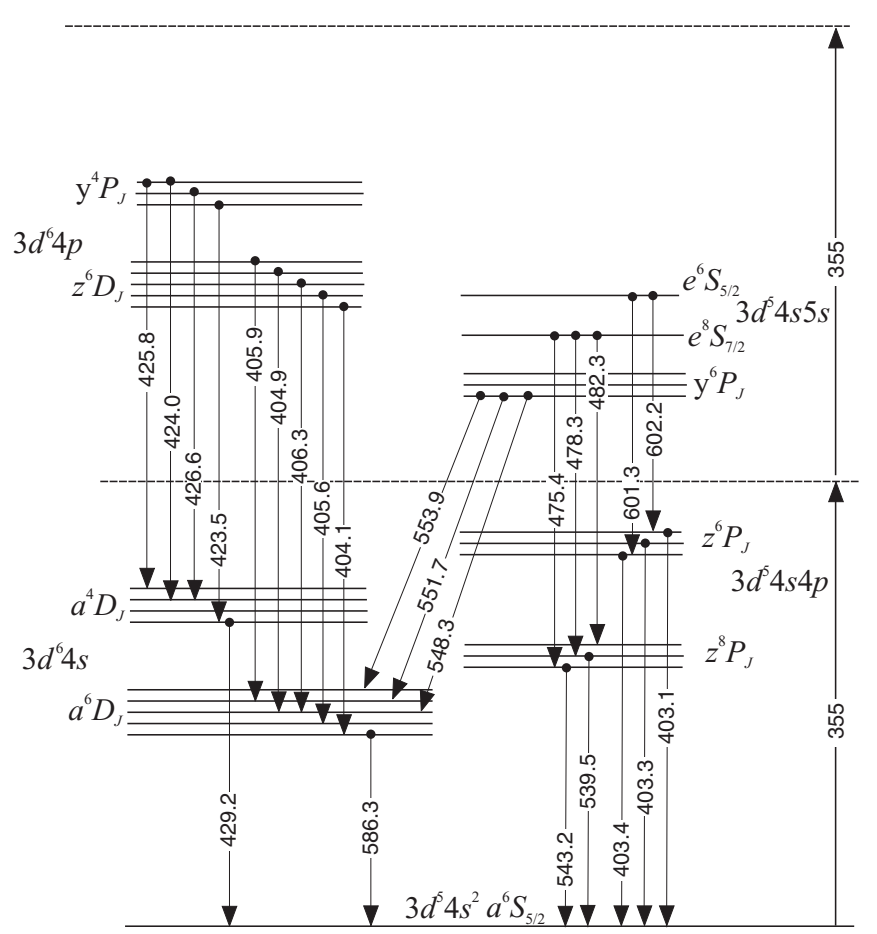

FIG. 1. Electronic states and transitions of $\mathrm{Mn}$ atom. ${ }^{8}$ Because of the very large number of excited states, only the states discussed in the present work are shown. Horizontal dashed lines indicate the energies accessible by onephoton and two-photon excitation at $\lambda=355 \mathrm{~nm}$. Transition wavelengths are given in $\mathrm{nm}$

We have also studied the dynamics of the luminescence decay following an exciting laser pulse. For this we recorded the laser-induced luminescence at a selected wavelength by means of a photomultiplier tube mounted at the exit slit of the spectrograph, and operated in the single-photon counting mode. The photon count rate was analyzed by an autocorrelator (Flex03LQ from Correlator.com) with a time-resolution of $10 \mathrm{~ns}$, yielding an autocorrelation function that reflects the dynamics of the photon-counting rate averaged over many excitation cycles.

\section{B. Luminescence spectra}

We first discuss the luminescence spectrum obtained by exciting the Mn-doped solid He sample with radiation from the pulsed, frequency tripled Nd:YAG laser $(\lambda=355 \mathrm{~nm})$. Absorption of this UV radiation efficiently populates some highly excited states of $\mathrm{Mn}$, thereby initiating a cascade of radiative and radiationless transitions towards the ground state. The wavelengths of the observed spectral lines and their assignments are collected in Table I.

We have identified in particular fluorescence from the $e^{6} S_{5 / 2}$ and $e^{8} S_{7 / 2}$ excited states which belong to the $3 d^{5} 4 s 5 s$ configuration and which decay to the $z^{6} P_{J}$ and $z^{8} P_{J^{\prime}}$ states of the $3 d^{5} 4 s 4 p$ configuration (Fig. 1). The observed spectrum of the $e^{8} S_{7 / 2}-z^{8} P_{5 / 2,7 / 2,9 / 2}$ triplet is shown in Fig. 2(a). The three lines are blueshifted by 93,97 , and $91 \mathrm{~cm}^{-1}$, respectively, with respect to the transitions in the free $\mathrm{Mn}$ atom and have FWHM spectral widths of $85-90 \mathrm{~cm}^{-1}$. The spectrum of the $e^{6} S_{5 / 2}-z^{6} P_{3 / 2,5 / 2,7 / 2}$ triplet is shown in Fig. 2(b). Due to the
TABLE I. Electronic transitions in Mn atoms observed in the laser-induced luminescence spectrum of Mn-doped solid He. Unresolved lines are marked by asterisks.

\begin{tabular}{|c|c|c|c|}
\hline & Transition & $\lambda_{\text {free }}(\mathrm{nm})$ & $\lambda_{\exp }(\mathrm{nm})$ \\
\hline \multirow[t]{5}{*}{$3 d^{5} 4 s 4 p-3 d^{5} 4 s^{2}$} & $z^{6} P_{7 / 2}-a^{6} S_{5 / 2}$ & 403.1 & $403^{*}$ \\
\hline & $z^{6} P_{5 / 2}-a^{6} S_{5 / 2}$ & 403.3 & $403^{*}$ \\
\hline & $z^{6} P_{3 / 2}-a^{6} S_{5 / 2}$ & 403.4 & $403^{*}$ \\
\hline & $z^{8} P_{7 / 2}-a^{6} S_{5 / 2}$ & 539.5 & 535.4 \\
\hline & $z^{8} P_{5 / 2}-a^{6} S_{5 / 2}$ & 543.2 & 537.8 \\
\hline \multirow[t]{18}{*}{$3 d^{6} 4 p-3 d^{6} 4 s$} & $z^{6} D_{7 / 2}-a^{6} D_{9 / 2}$ & 401.8 & $408^{*}$ \\
\hline & $z^{6} D_{9 / 2}-a^{6} D_{9 / 2}$ & 404.1 & $408^{*}$ \\
\hline & $z^{6} D_{3 / 2}-a^{6} D_{5 / 2}$ & 404.9 & $408^{*}$ \\
\hline & $z^{6} D_{7 / 2}-a^{6} D_{7 / 2}$ & 405.6 & $408^{*}$ \\
\hline & $z^{6} D_{1 / 2}-a^{6} D_{3 / 2}$ & 405.9 & $408^{*}$ \\
\hline & $z^{6} D_{5 / 2}-a^{6} D_{5 / 2}$ & 406.3 & $408^{*}$ \\
\hline & $z^{6} D_{3 / 2}-a^{6} D_{3 / 2}$ & 406.8 & $408^{*}$ \\
\hline & $z^{6} D_{1 / 2}-a^{6} D_{1 / 2}$ & 407.0 & $408^{*}$ \\
\hline & $z^{6} D_{3 / 2}-a^{6} D_{1 / 2}$ & 407.9 & $408^{*}$ \\
\hline & $z^{6} D_{5 / 2}-a^{6} D_{3 / 2}$ & 408.3 & $408^{*}$ \\
\hline & $z^{6} D_{7 / 2}-a^{6} D_{5 / 2}$ & 408.4 & $408^{*}$ \\
\hline & $y^{4} P_{5 / 2}-a^{4} D_{7 / 2}$ & 423.5 & $422.5^{*}$ \\
\hline & $y^{4} P_{1 / 2}-a^{4} D_{3 / 2}$ & 424.0 & $422.5^{*}$ \\
\hline & $y^{4} P_{1 / 2}-a^{4} D_{1 / 2}$ & 425.8 & $425.5^{*}$ \\
\hline & $y^{4} P_{3 / 2}-a^{4} D_{3 / 2}$ & 426.6 & $425.5^{*}$ \\
\hline & $y^{4} P_{5 / 2}-a^{4} D_{5 / 2}$ & 428.1 & $425.5^{*}$ \\
\hline & $y^{4} P_{3 / 2}-a^{4} D_{1 / 2}$ & 428.4 & $425.5^{*}$ \\
\hline & $y^{4} P_{5 / 2}-a^{4} D_{3 / 2}$ & 431.3 & $?$ \\
\hline \multirow[t]{6}{*}{$3 d^{5} 4 s 5 s-3 d^{5} 4 s 4 p$} & $e^{8} S_{7 / 2}-z^{8} P_{5 / 2}$ & 475.4 & 473.3 \\
\hline & $e^{8} S_{7 / 2}-z^{8} P_{7 / 2}$ & 478.3 & 476.1 \\
\hline & $e^{8} S_{7 / 2}-z^{8} P_{9 / 2}$ & 482.3 & 480.2 \\
\hline & $e^{6} S_{5 / 2}-z^{6} P_{3 / 2}$ & 601.3 & $596^{*}$ \\
\hline & $e^{6} S_{5 / 2}-z^{6} P_{5 / 2}$ & 601.6 & $596^{*}$ \\
\hline & $e^{6} S_{5 / 2}-z^{6} P_{7 / 2}$ & 602.2 & $596^{*}$ \\
\hline \multirow[t]{2}{*}{$3 d^{6} 4 s-3 d^{5} 4 s^{2}$} & $a^{6} D_{9 / 2}-a^{6} S_{5 / 2}$ & 586.3 & 586.4 \\
\hline & $a^{4} D_{7 / 2}-a^{6} S_{5 / 2}$ & 429.2 & 429.1 \\
\hline
\end{tabular}

small fine structure splitting of the $z^{6} P_{J}$ states $\left(9\right.$ and $14 \mathrm{~cm}^{-1}$ in the free atom), the three lines strongly overlap and cannot be resolved. The observed spectral feature has a FWHM spectral width of $110 \mathrm{~cm}^{-1}$ and its maximum is blueshifted by 148 $\mathrm{cm}^{-1}$ with respect to the central triplet component in the free atom.

The $z^{6} P_{J}$ and $z^{8} P_{J^{\prime}}$ states decay to the $3 d^{5} 4 s^{2} a^{6} S_{5 / 2}$ ground state. The $z^{8} P_{5 / 2,7 / 2}-a^{6} S_{5 / 2}$ doublet (strongly overlapping lines) is shown in Fig. 3(a). In order to obtain the spectral widths and shifts of the two doublet components, we have fitted the observed spectrum with a sum of two Gaussians. The two lines are found to be blueshifted with respect to the free-atomic transitions by 150 and $180 \mathrm{~cm}^{-1}$, respectively, and to have spectral widths of 102 and $98 \mathrm{~cm}^{-1}$, respectively. The close-lying fine-structure components can be strongly mixed by the interaction with the matrix, leading to a quenching of the upper substates. In matrix-isolation studies, one therefore usually observes only luminescence from the lowest component in each multiplet. It is thus surprising that both fine-structure components of the $z^{8} P_{5 / 2,7 / 2}-a^{6} S_{5 / 2}$ doublet are observed here with nearly equal amplitudes. It is possible that one of the two observed spectral components belongs to a different transition with a close-lying wavelength. 


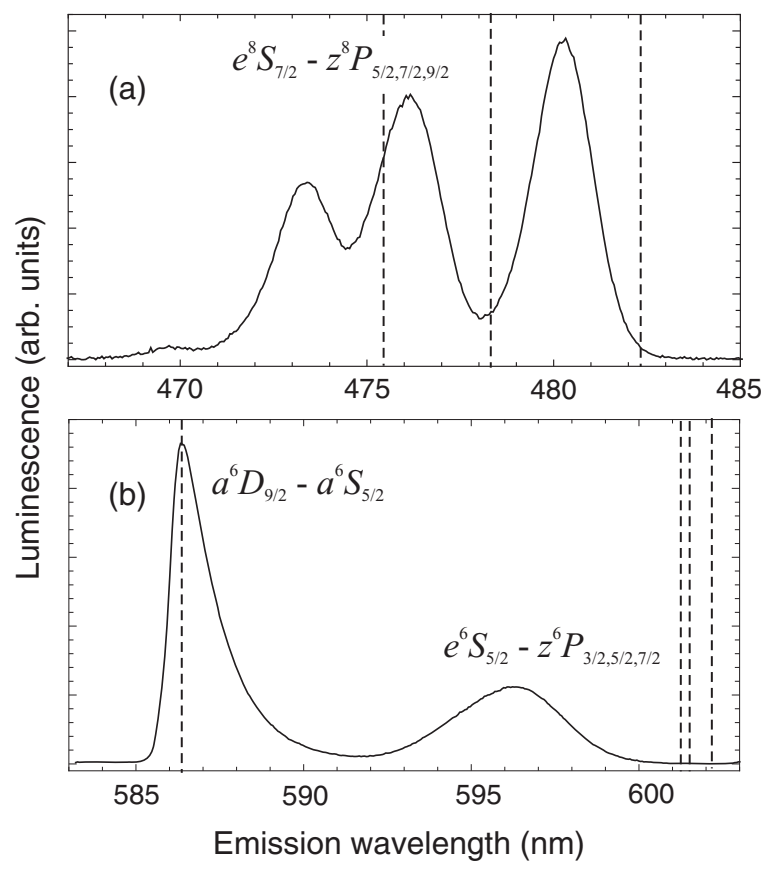

FIG. 2. Laser induced luminescence spectra of Mn atoms in solid He following excitation at $355 \mathrm{~nm}$. The vertical dashed lines indicate the transition wavelengths in the free $\mathrm{Mn}$ atom. (a) $3 d^{5} 4 s 5 s e^{8} S_{7 / 2}-3 d^{5} 4 s 4 p z^{8} P_{5 / 2,7 / 2,9 / 2}$ fine-structure triplet. (b) $3 d^{5} 4 s 5 s e^{6} S_{5 / 2}-3 d^{5} 4 s 4 p z^{6} P_{3 / 2,5 / 2,7 / 2}$ transitions and the forbidden $3 d^{6} 4 s a^{6} D_{9 / 2}-3 d^{5} 4 s^{2} a^{6} S_{5 / 2}$ transition.

The closest allowed transition is $3 d^{5} 4 s 4 p y^{6} P_{3 / 2}-3 d^{6} 4 s a^{6} D_{5 / 2}$ at $548.3 \mathrm{~nm}$. Assigning the shorter-wavelength component of the doublet to the $z^{8} P_{5 / 2}-a^{6} S_{5 / 2}$ transition and the longerwavelength component to the $y^{6} P_{3 / 2}-a^{6} D_{5 / 2}$ transition, we determine their line shifts to be 278 and $347 \mathrm{~cm}^{-1}$, respectively. However, in this case we would expect to observe also two other transitions from the $y^{6} P_{3 / 2}$ state (the lowest in the $y^{6} P_{J}$ multiplet) towards the $a^{6} D_{3 / 2}$ and $a^{6} D_{1 / 2}$ states (551.7 and $553.9 \mathrm{~nm}$ in the free atom).

The components of the $z^{6} P_{3 / 2,5 / 2,7 / 2}-a^{6} S_{5 / 2}$ triplet cannot be resolved by our spectrometer. Their spectrum is shown in Fig. 4(a). The peak is blueshifted by $41 \mathrm{~cm}^{-1}$ with respect to the lowest component of the triplet in the free $\mathrm{Mn}$ atom and has a spectral width of $93 \mathrm{~cm}^{-1}$.

A significant part of the excited state population is transferred to the metastable low-lying $a^{6} D_{J}$ and $a^{4} D_{J^{\prime}}$ states that belong to the $3 d^{6} 4 s$ configuration. We observe a strong luminescence signal on the two forbidden transitions originating from the lowest state of each group. The $a^{6} D_{9 / 2}-a^{6} S_{5 / 2}$ line is observed at $586 \mathrm{~nm}$ as shown in Fig. 2(b), while Fig. 4(b) shows the $a^{4} D_{7 / 2}-a^{6} S_{5 / 2}$ line at $429 \mathrm{~nm}$. Both transitions have asymmetric lineshapes with extended red wings. They are practically not shifted with respect to the free-atomic transitions and their FWHM spectral widths are 42 and $38 \mathrm{~cm}^{-1}$, respectively.

The $a^{6} D_{J}$ states lie approximately $800 \mathrm{~cm}^{-1}$ below the $z^{8} P_{J}$ states (see Fig. 1) and can be populated by radiationless transitions from that multiplet. Similarly, the $a^{4} D_{J}$ states lie $\approx 1000 \mathrm{~cm}^{-1}$ below the $z^{6} P_{J}$ multiplet and can be populated by radiationless transitions from the latter. There exists a further population channel starting from highly excited states of

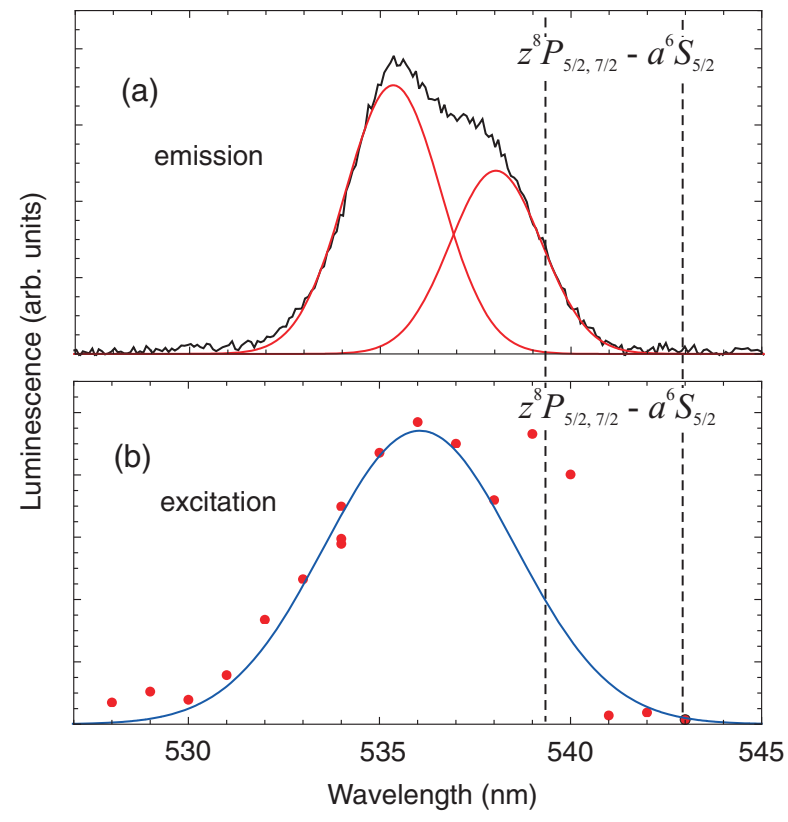

FIG. 3. Emission (a) and excitation (b) spectra of the $3 d^{5} 4 s 4 p z^{8} P_{J}-3 d^{5} 4 s^{2}$ $a^{6} S_{5 / 2}$ transitions of $\mathrm{Mn}$ in solid He. The vertical dashed lines indicate the wavelengths of the $z^{8} P_{5 / 2,7 / 2}-a^{6} S_{5 / 2}$ fine-structure doublet in the free $\mathrm{Mn}$ atom. The luminescence in (a) is excited by the third harmonic of the Nd:YAG laser $(355 \mathrm{~nm})$

the $3 d^{6} 4 p$ configuration. The two small peaks at 422.6 and $425.7 \mathrm{~nm}$ of Fig. 4(b) can be assigned to the group of $3 d^{6} 4 p$ $y^{4} P_{J}-3 d^{6} 4 s a^{4} D_{J^{\prime}}$ transitions. In total, this group consists of the 7 transitions listed in Table I. Assuming that the two higher-lying substates of the $y^{4} P_{J}$ triplet are quenched towards the lowest substate, we can assign the two observed peaks to the two transitions from $y^{4} P_{5 / 2}$ state to the $a^{4} D_{7 / 2}$ and $a^{4} D_{5 / 2}$ states, blueshifted by 57 and $139 \mathrm{~cm}^{-1}$, respectively and having spectral widths of 75 and $95 \mathrm{~cm}^{-1}$. The third transition originating from the $y^{4} P_{5 / 2}$ state is significantly weaker and probably overlaps with the much stronger $a^{4} D_{7 / 2}-a^{6} S_{5 / 2}$ line. The large difference in the spectral shifts and widths of the two peaks suggests that they in fact contain contributions from transition originating from all three $y^{4} P_{J}$ states (see Table I).

Similarly, the broad spectral feature in Fig. 4(a) centered at $408 \mathrm{~nm}$ can be tentatively assigned to the unresolved $3 d^{6} 4 p$ $z^{6} D_{J}-3 d^{6} 4 s a^{6} D_{J^{\prime}}$ multiplet. If this assignment is correct, the transitions are redshifted with respect to the free-atomic wavelengths (see Table I).

As shown in Fig. 1, the observed luminescence cascades originate from the states $e^{8} S_{7 / 2}\left(39431 \mathrm{~cm}^{-1}\right), e^{6} S_{5 / 2}$ $\left(41404 \mathrm{~cm}^{-1}\right)$ and possibly from $y^{6} P_{J}\left(35690-35770 \mathrm{~cm}^{-1}\right)$, $z^{6} D_{J}\left(41789-42199 \mathrm{~cm}^{-1}\right)$, and $y^{4} P_{J}\left(46901-47299 \mathrm{~cm}^{-1}\right)$. All these states are not accessible by single-photon absorption at $\lambda=355 \mathrm{~nm}\left(28170 \mathrm{~cm}^{-1}\right)$, but are accessible by two-photon excitation. We therefore suggest that the excitation proceeds via a quasi-resonant two-photon absorption towards some higher-lying excited state that is quenched by radiationless transitions towards the emitting luminescence states. Alternatively, it may involve a 3-photon photoionization of the Mn atom (ionization threshold $59959 \mathrm{~cm}^{-1}$ in 


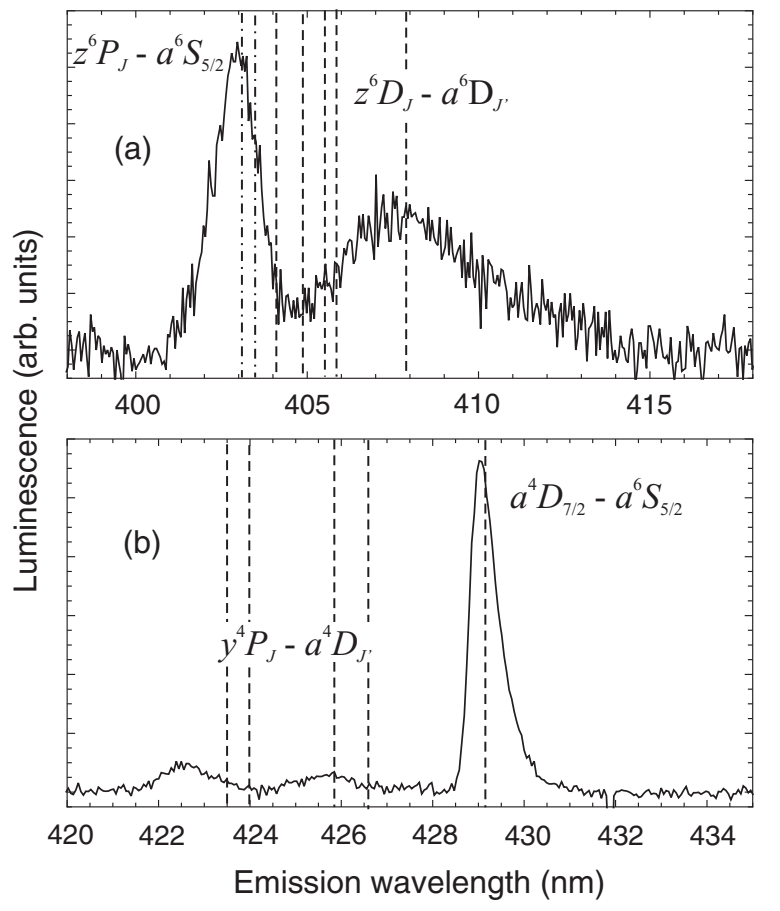

FIG. 4. Laser-induced luminescence spectra of $\mathrm{Mn}$ atoms in solid $\mathrm{He}$ following excitation at $355 \mathrm{~nm}$. The vertical dashed and dot-dashed lines indicate the transition wavelengths in the free Mn atom. (a) Unresolved lines of the $3 d^{5} 4 s 4 p z^{6} P_{J}-3 d^{5} 4 s^{2} a^{6} S_{5 / 2}$ and $3 d^{6} 4 p z^{6} D_{J}-3 d^{6} 4 s a^{6} D_{J^{\prime}}$ transitions. (b) Forbidden $3 d^{6} 4 s a^{4} D_{7 / 2}-3 d^{5} 4 s^{2} a^{6} S_{5 / 2}$ transition and a group of unresolved $3 d^{6} 4 p y^{4} P_{J}-3 d^{6} 4 s a^{4} D_{J^{\prime}}$ transitions.

the free atom) and a subsequent recombination that populates the highly excited states. The available experimental data do not allow us to identify the intermediate states involved in the excitation process. A similar situation was encountered in our recent experiments with $\mathrm{Ba}^{5} \mathrm{Cu}^{4}$ and $\mathrm{Au}^{6}$ atoms in solid He.

\section{Excitation spectrum of the $a^{6} S_{5 / 2}-z^{8} P_{J}$ transitions}

Among all observed excited states of the Mn atom, only the $3 d^{5} 4 s 4 p z^{8} P_{5 / 2,7 / 2}$ states possess strong transitions to the ground state that are accessible with our tunable OPO system. We have investigated their resonant excitation spectra by tuning the OPO over the range of 525-545 nm and monitoring the luminescence yield. Both $z^{8} P_{5 / 2,7 / 2}$ states are strongly quenched and their population is transferred to the metastable $3 d^{6} 4 s a^{6} D_{9 / 2}$ state, i.e., the lowest-lying excited state of the Mn atom. A similar quenching effect was observed earlier in experiments with $\mathrm{Ba}, \mathrm{Cu}$ and alkali dimer molecules. ${ }^{4}$ Figure 3(b) shows the luminescence signal measured at the wavelength of the forbidden $a^{6} D_{9 / 2}-a^{6} S_{5 / 2}$ transition at 586 $\mathrm{nm}$ as a function of the OPO wavelength.

It is expected that the two components of the $a^{6} S_{5 / 2}$ - $z^{8} P_{5 / 2,7 / 2}$ doublet in absorption show a broader line than in emission and therefore cannot be resolved. We therefore fit our data points with a single Gaussian peaked at $536.1 \mathrm{~nm}$. The maximum is blueshifted by 124 and $253 \mathrm{~cm}^{-1}$ with respect to the two fine-structure components in the free atom. The FWHM spectral width is $205 \mathrm{~cm}^{-1}$.

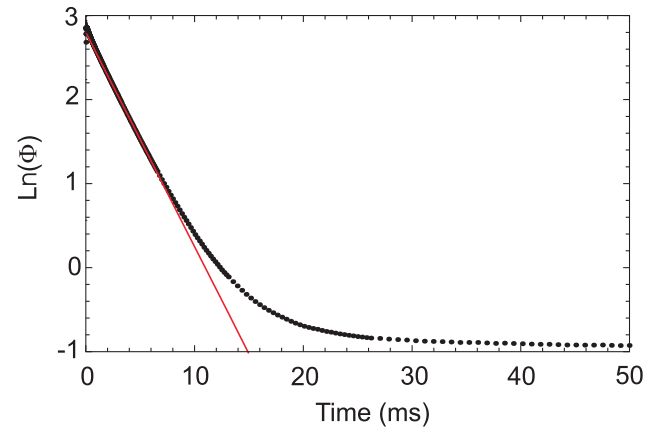

FIG. 5. Autocorrelation function of the luminescence signal at $\lambda=586 \mathrm{~nm}$ following an excitation pulse at $t=0$. Dots: experimental data; solid line: fitted exponential decay.

\section{Dynamics of the $a^{6} D_{9 / 2}-a^{6} S_{5 / 2}$ transition}

We have studied the decay time of the long-lived metastable $3 d^{6} 4 s a^{6} D_{9 / 2}$ state by measuring the autocorrelation function $\Phi(t)$ of the luminescence signal at the $a^{6} D_{9 / 2}$ - $a^{6} S_{5 / 2}$ transition. A typical recording of $\Phi(t)$ is represented in Fig. 5 on a semi-logarithmic scale. The atoms were excited by nanosecond laser pulses at $\lambda=355 \mathrm{~nm}$, fired at a rate of $10 \mathrm{~Hz}$. The observed luminescence at $\lambda=586 \mathrm{~nm}$ decays within several milliseconds after the excitation pulse, before the arrival of the next pulse. In the accessible range of delay times, $\tau=10^{-5}-10^{-1} \mathrm{~s}$, the decrease of the autocorrelation function is due to the decay of the luminescence amplitude.

The data presented in Fig. 5 do not represent a single exponential decay, but rather the sum of at least two decay processes. However, we cannot unambiguously assign the second (slow) decay to any physical process nor to imperfections in the detection system. We therefore only consider the dominant fast decay time and attribute it to the effective lifetime of the luminescent state, or at least its lower limit.

We have fitted the observed decay curves with an exponential function and obtain characteristic decay times $\tau_{d}=3.9 \mathrm{~ms}$. This is approximately 900 times faster than the radiative lifetime $\tau_{r}=3.4 \mathrm{~s}^{14}$ of the $a^{6} D_{9 / 2}$ state in the free Mn atom, but slightly slower than typical decay times measured in heavier rare-gas matrices. ${ }^{10,11}$

\section{DISCUSSION}

\section{A. Outer-shell transitions}

In the present experiment, we have observed several electronic transitions between the $4 s$ and $4 p$ shells as well as between the $4 p$ and $5 s$ shells. These outer-shell transitions are broadened by $85-100 \mathrm{~cm}^{-1}$ and blueshifted with respect to the free-atomic lines by some $100-180 \mathrm{~cm}^{-1}$. Only the $z^{6} P_{J}$ - $a^{6} S_{5 / 2}$ spectrum has a significantly smaller blueshift and the tentatively assigned $z^{6} D_{J}-a^{6} D_{J^{\prime}}$ multiplet seems to be redshifted. The lineshapes (at least those that we were able to resolve) are symmetric and very close to Gaussians. All mentioned features closely resemble those of outer-shell transitions of other atomic species, such as $\mathrm{Cs}, \mathrm{Ba}$, or $\mathrm{Cu}$ in solid He matrices. ${ }^{4,5,15}$ 
The comparison of the excitation and luminescence spectra of the $a^{6} S_{5 / 2}-z^{8} P_{J}$ doublet (Fig. 3) demonstrates very similar lineshifts for both spectra. This observation is in contrast with the trend observed in the spectra of outer-shell transitions of alkali and alkali-earth metals, for which the lineshift in absorption is significantly larger than that in emission. As discussed below, in Sec. III C, the spectra of the outer shell transitions are usually well described by the atomic bubble model which also predicts a large difference between the excitation and emission lineshifts. In the absence of accurate $\mathrm{Mn}-\mathrm{He}$ interaction potentials, we cannot suggest any plausible explanation for the observed anomaly.

The spectra of the $z^{6} P_{J}-a^{6} S_{5 / 2}$ and $z^{8} P_{J}-a^{6} S_{5 / 2}$ transitions can be compared with those obtained in heavier raregas matrices..$^{9,10}$ The luminescence spectra observed in Ar, $\mathrm{Kr}$, and $\mathrm{Xe}$ matrices are shifted towards lower energies by $600-800 \mathrm{~cm}^{-1}$ and broadened by $\approx 500 \mathrm{~cm}^{-1}$.

It is thus clear that the electronic states of $\mathrm{Mn}$ in heavier rare-gas solids undergo a much stronger perturbation than in solid He matrices. As discussed in Ref. 13, a similar behavior is observed with alkali-metal atoms, and the stronger perturbation in the heavier rare-gas crystals can be attributed to their larger lattice stiffness compared to solid He. The higher stiffness produces much tighter trapping sites with symmetries determined by the crystalline structure, while in the softer solid He matrix, the metal atoms reside in bubbles whose size and shape are mostly determined by the dopants' electronic density distribution.

\section{B. Inter-shell transitions}

The main motivation for the present study was to obtain a deeper insight into the $3 d^{6} 4 s-3 d^{5} 4 s^{2}$ inter-shell transitions of Mn. They clearly differ from the outer-shell transitions in so far as they are practically not shifted with respect to the free-atomic lines, they have smaller spectral widths and they are non-symmetric with red wings that are more extended than the blue wings. The differences in the linewidths and the lineshifts are attributed to a screening effect. ${ }^{1-3}$ The electrons from the inner-shells are much less perturbed by the interaction with helium since they are screened by the valence electron density that repels the He atoms.

Similar features were observed before in the luminescence spectrum of the $5 d 6 s^{1} D_{2}-6 s^{2}{ }^{1} S_{0}$ inter-shell transition of $\mathrm{Ba}$ in solid He. ${ }^{5}$ The latter also shows a strongly asymmetric lineshape with a FWHM linewidth of $40 \mathrm{~cm}^{-1}$ that is not shifted with respect to the free-atomic wavelength.

The inter-shell transitions of $\mathrm{Mn}$ in solid He can also be compared to the $3 d^{9} 4 s^{2}-3 d^{10} 4 s$ transitions of $\mathrm{Cu}$ and the $5 d^{9} 6 s^{2}-5 d^{10} 6 s$ transition of Au. ${ }^{1,4}$ These three lines of $\mathrm{Cu}$ and $\mathrm{Au}$ belong to the same class of transitions between the outer $n s$ shell and the inner $(n-1) d$ shell. However, their lineshapes differ completely from the inter-shell transitions of $\mathrm{Ba}$ and Mn. They consist of two spectral components: a sharp zerophonon line (ZPL) and a broader phonon wing (PW) that is redshifted with respect to the ZPL. The linewidth of the ZPL is limited by our spectrometer resolution, and the FWHM of the $\mathrm{PW}$ is $\approx 20 \mathrm{~cm}^{-1}$, i.e., approximately two times smaller

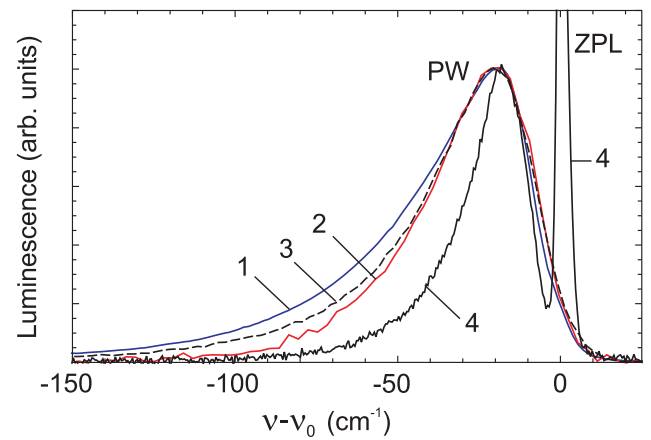

FIG. 6. Comparison of the lineshapes of different inter-shell transitions in $\mathrm{Mn}, \mathrm{Cu}$, and $\mathrm{Ba}$ atoms in solid He. Line 1: $a^{6} D_{9 / 2}-a^{6} S_{5 / 2}$ transition of $\mathrm{Mn}$ $(\lambda=586 \mathrm{~nm})$, line 2: $a^{4} D_{7 / 2}-a^{6} S_{5 / 2}$ transition in $\mathrm{Mn}(\lambda=429 \mathrm{~nm})$, line 3: $5 d 6 s{ }^{1} D_{2}-6 s^{2}{ }^{1} S_{0}$ transition of $\mathrm{Ba}(\lambda=878 \mathrm{~nm})$, and line $4-3 d^{9} 4 s^{2}{ }^{2} D_{5 / 2}$ - $3 d^{10} 4 s^{2} S_{1 / 2}$ transition in $\mathrm{Cu}(\lambda=894 \mathrm{~nm})$.

than the spectral widths of the inter-shell lines in $\mathrm{Mn}$ and $\mathrm{Ba}$. In Fig. 6, the lineshapes of the three inter-shell transitions of $\mathrm{Mn}$ and $\mathrm{Ba}$ are compared to the lineshape of the $3 d^{9} 4 s^{2}{ }^{2} D_{5 / 2}$ - $3 d^{10} 4 s^{2} S_{1 / 2}$ transition's phonon wing in $\mathrm{Cu}$. All four lineshapes are represented on the same energy scale and we have shifted the lines so that their maxima coincide. One can see that the PW lineshape of $\mathrm{Cu}$ has an even stronger asymmetry than the lines of $\mathrm{Mn}$ and $\mathrm{Ba}$.

\section{Qualitative model}

The origin of the pronounced difference between the observed inter-shell lineshapes of $\mathrm{Cu}$ and $\mathrm{Au}$ on one hand, and the inter-shell transitions in $\mathrm{Mn}$ and $\mathrm{Ba}$ on the other hand is not clear. As discussed in Ref. 13, the lineshape of an electronic transition in an atom trapped in a bubble in condensed $\mathrm{He}$ is determined by the quantum fluctuations of the bubble size. Transition energies are determined by the relative positions (and shapes) of the potentials describing the total energy of the atom-bubble system, while the widths and asymmetries of the spectral lines are determined by the slope and curvature of the final state's potential curves (Fig. 7). The three observed types of lineshapes described above can be understood qualitatively based on the simple line broadening mechanism shown in Fig. 7, in which we represent the total energy of the atom-bubble system as a function of the bubble radius

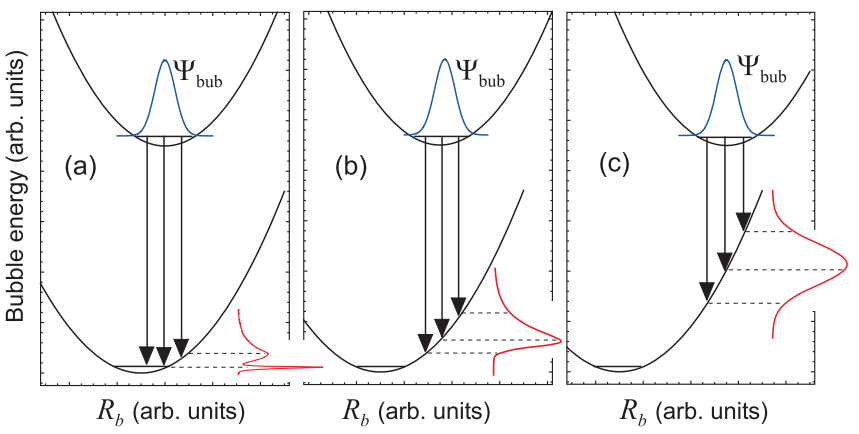

FIG. 7. Electronic transitions between vibration states of an atom in atomic bubble. (a) Inner-shell or inter-shell transition with a small change of the bubble radius; (b) inter-shell transition with intermediate change of bubble radius; and (c) outer-shell transition with a large change of the bubble radius. 
$R_{b}$ for the upper and lower electronic states involved in the transition. The quantum uncertainty of the upper state's bubble radius is described by the Gaussian probability distribution $\left|\Psi_{\text {bub }}\left(R_{b}\right)\right|^{2}$ that represents the zero-point vibration of the equilibrium bubble that is reached following excitation of the upper electronic state and vibrational relaxation within that state. The luminescence lineshape can then be obtained by calculating the weighted projection of $\left|\Psi_{\text {bub }}\left(R_{b}\right)\right|^{2}$ onto the lower states's potential as shown in the figure.

In Ref. 1, we have discussed the two limiting cases corresponding to Figs. 7(a) and 7(c), respectively. When the minima of the two potential curves lie approximately at the same bubble radius as shown on Fig. 7(a), there is a strong probability for a luminescence transition from the upper state' zero-vibration level to the zero-vibration level of the lower electronic state. The transition will leave the bubble radius unchanged and therefore produce no phonons in the matrix, so that its spectrum will consist of a sharp peak, the zerophonon line. Transitions towards other vibronic states produce a broader and red-shifted spectral feature, the phonon wing. The transitions $(n-1) d^{9} n s^{2}-(n-1) d^{10} n s$ in $\mathrm{Cu}$ and Au belong to this type as well as the $4 f^{7} 6 s^{2}-4 f^{6} 5 d 6 s^{2}$ transitions of Eu, ${ }^{3}$ the $4 f^{13} 6 s^{2}-4 f^{12} 5 d 6 s^{2}$ transition of $\mathrm{Tm},{ }^{2}$ and a transition within the $5 d^{9} 6 s^{2}$ configuration of Au. ${ }^{1}$

Figure $7(\mathrm{c})$ shows the other limiting case in which the minima of the two potential curves are strongly shifted with respect to each other, thus representing atoms with a considerably larger equilibrium bubble in the upper than in the lower state. The luminescence transition then takes the atom from the minimum of the upper potential curve to the steep part of the lower potential curve that can be approximated by a straight line. As a result, one obtains a very broad and symmetric lineshape, a feature that is typical for the valence electron transitions in alkali, alkaline-earth, and transition-metal atoms. ${ }^{4,5,13}$

The $3 d^{6} 4 s-3 d^{5} 4 s^{2}$ transitions of Mn observed in the present work and the $5 d 6 s-6 s^{2}$ transition of Ba studied in Ref. 5 represent an intermediate case, such as shown in Fig. 7(b). The shift of the minima of the two potential curves is too large for the ZPL to be observed. On the other hand, it is not as large as in the case of valence electron transitions. The potential curve of the lower electronic state close to the minimum has a large curvature that results in a strong asymmetry of the luminescence spectrum.

The intra-shell transitions of $\mathrm{Mn}$ in heavier rare-gas matrices have been successfully analyzed ${ }^{10,11}$ by fitting the so-called $W_{p}$ spectral function. ${ }^{16}$ In particular, this model provides estimates of the Huang-Rhys parameter $S$ that characterizes the shift between the upper and lower potential curves in units of the characteristic phonon frequency, $E_{p h}$. We have also tried to apply an analysis in terms of the $W_{p}$ lineshape function $^{16}$ to our experimental spectra and could not obtain a good fit. First of all, the position of the zero-phonon line (ZPL) cannot be guessed from our data with sufficient accuracy and has to be taken as one of the fit parameters. Second, the value of characteristic phonon frequency, $E_{p h}$ $\left(21 \mathrm{~cm}^{-1}\right)$ resulting from a best fit is several times larger than the typical frequencies of atomic bubble vibrations reported earlier for Cs $\left(5 \mathrm{~cm}^{-1}\right.$, Ref. 15$), \mathrm{Ba}\left(4 \mathrm{~cm}^{-1}\right.$, Ref. 17), and
$\mathrm{Cu}\left(10 \mathrm{~cm}^{-1}\right.$, Ref. 1). A variation of $E_{p h}$ from 10 to $30 \mathrm{~cm}^{-1}$ does not lead to a significant deterioration of the fit and results in variations of the Huang-Rhys parameter $S$ between 4.0 and 0.6. It is thus difficult to obtain a reliable value for the $S$ parameter that could be used for comparing spectra from different matrices. Finally, the typical asymmetric lineshape with a rather sharp peak that is observed experimentally (see Fig. 6) cannot be well reproduced by $W_{p}$. The unsatisfactory results of such fits probably indicate that the assumptions underlying the $W_{p}$ model are not appropriate for solid He which is a quantum crystal and whose properties therefore differ significantly from those of the heavier rare-gas solids.

\section{Comparison to heavier noble gas matrices}

It is interesting to compare the $3 d^{6} 4 s-3 d^{5} 4 s^{2}$ spectra of $\mathrm{Mn}$ in solid He with those observed in the heavier rare-gas matrices Ar, Kr, and Xe. ${ }^{10,11}$ The $a^{4} D_{7 / 2}-a^{6} S_{5 / 2}$ transition has been observed ${ }^{10}$ only in the luminescence spectrum and only in $\mathrm{Ar}$ and $\mathrm{Kr}$ matrices. In both matrices, it displays a rich spectral structure with an overall width of $\approx 100 \mathrm{~cm}^{-1}$ as well as a pronounced ZPL. For the $a^{6} D_{9 / 2}-a^{6} S_{5 / 2}$ transition, both excitation and luminescence spectra are available. ${ }^{11}$ In $\mathrm{Kr}$, the luminescence line is $\approx 100 \mathrm{~cm}^{-1}$ broad and has a rich substructure that includes a ZPL. In Ar, the luminescence lineshape has a similar width but shows no ZPL, and in Xe the line is significantly broader. The $a^{6} D_{9 / 2}-a^{6} S_{5 / 2}$ transition is thus perturbed stronger than the $a^{4} D_{7 / 2}-a^{6} S_{5 / 2}$ transition and the perturbation is larger in Ar than in $\mathrm{Kr}$. It was also found ${ }^{12}$ that the crystal field splitting leads to multiple zero-phonon lines.

It seems that the solid He matrix exerts a stronger perturbation on the forbidden $3 d^{6} 4 s-3 d^{5} 4 s^{2}$ transitions of Mn than solid Ar and $\mathrm{Kr}$ matrices. This observation is surprising since so far He matrices, because of their softness, have been considered to be the least perturbing matrices of all solid noble gases. ${ }^{13}$ As discussed above, in Sec. III A, the outer-shell transitions of Mn also support this general trend. Only the two inter-shell transitions demonstrate the anomaly.

Lacking the quantitative theory, we propose the following speculation on the origin of this anomalous behavior. The authors of Refs. 10-12 suggest that their inter-shell spectra correspond to Mn atoms occupying tetra-vacancy trapping sites in the matrices. These are rather large voids in the rigid crystalline lattices of $\mathrm{Ar}, \mathrm{Kr}$, and $\mathrm{Xe}$, whose shape is not altered by the interaction with the dopant and leads to a characteristic (small) splitting of the electronic states of the Mn. When the Mn atoms undergoes an inter-shell transition, the change of the electronic density distribution is rather small and does not lead to any rearrangement of the rare-gas atoms forming the cage. Thus, the phonon creation probability is very low and the spectrum has a pronounced ZPL structure with a splitting that represents the trapping site symmetry. In solid He, the crystalline lattice is very much softer and the He atoms in the vicinity of the dopant are completely rearranged to minimize the void (atomic bubble). The dopant thus locally imposes its own symmetry on the matrix. Even a small change of the 
electronic density distribution of the dopant leads to a corresponding modification of the atomic bubble shape and size and thus generates a number of phonons. This would explain why no ZPL could be observed in the luminescence spectrum of $\mathrm{Mn}$ in $\mathrm{He}$.

\section{SUMMARY}

In summary, we have carried out a spectroscopic study of manganese atoms isolated in a solid helium matrix. The luminescence spectra of the valence electron transitions in manganese behave very similarly to the resonance lines of the alkali, alkali-earth, and coinage metals, displaying structureless lineshapes with a large broadening and a large blueshift. On the other hand, the resonances of inter-shell transitions in Mn are practically not shifted and less broad. Nevertheless, we were not able to observe any spectral structure that might be interpreted as a zero-phonon line. This fact is surprising when considering that ZPLs have been detected recently in the excitation and fluorescence spectra of the same Mn transitions with manganese atoms isolated in solid $\mathrm{Ar}$ and $\mathrm{Kr}$. We propose a qualitative interpretation of these observations.

Only one valence electron transition, the $a^{6} S_{5 / 2}-z^{8} P_{5 / 2,7 / 2}$ doublet was studied also in absorption. Its excitation spectrum is blueshifted similar to the emission spectrum. That is in contrast with the spectra of other metals studied up to date, for which the absorption is shifted stronger than the emission.

\section{ACKNOWLEDGMENTS}

This work was supported by Grant No. 200020-119786 of the Schweizerischer Nationalfonds. P.M. acknowledges financial support from the Light and Matter (LiMat) project. We acknowledge help from F. Cardinaux and F. Scheffold, who provided the autocorrelator for the time-resolved study.

${ }^{1}$ P. Moroshkin, V. Lebedev, and A. Weis, Europhys. Lett. 96, 26002 (2011).

${ }^{2}$ K. Ishikawa, A. Hatakeyama, K. Gosyono-o, S. Wada, Y. Takahashi, and T. Yabuzaki, Phys. Rev. B 56, 780 (1997).

${ }^{3}$ Q. Hui and M. Takami, J. Low Temp. Phys. 119, 393 (2000).

${ }^{4}$ P. Moroshkin, V. Lebedev, and A. Weis, Phys. Rev. A 84, 052519 (2011).

${ }^{5}$ V. Lebedev, P. Moroshkin, and A. Weis, Phys. Rev. A 84, 022502 (2011).

${ }^{6}$ P. Moroshkin, V. Lebedev, and A. Weis, J. Chem. Phys. 139, 104307 (2013).

${ }^{7}$ J. P. Toennies and A. F. Vilesov, Angew. Chem.: Int. Ed. 43, 2622 (2004).

${ }^{8}$ W. F. Meggers, C. H. Corliss, and B. F. Scribner, Tables of Spectral Line Intensities (National Bureau of Standards, U.S., 1975).

${ }^{9}$ M. A. Collier and J. G. McCaffrey, J. Chem. Phys. 122, 054503 (2005).

${ }^{10}$ M. A. Collier and J. G. McCaffrey, J. Chem. Phys. 122, 184507 (2005).

${ }^{11}$ M. A. Collier, M. C. Ryan, and J. G. McCaffrey, J. Chem. Phys. 123, 044508 (2005).

${ }^{12}$ M. A. Collier, O. Byrne, C. Murray, and J. G. McCaffrey, J. Chem. Phys. 132, 164512 (2010).

${ }^{13}$ P. Moroshkin, A. Hofer, and A. Weis, Phys. Rep. 469, 1 (2008).

${ }^{14}$ H. Nussbaumer and J. P. Swings, Astrophys. J. 172, 121 (1972).

${ }^{15}$ A. Hofer, P. Moroshkin, S. Ulzega, D. Nettels, R. Müller-Siebert, and A. Weis, Phys. Rev. A 76, 022502 (2007).

${ }^{16} \mathrm{C}$. W. Struck and W. H. Fonger, Understanding Luminescence Spectra and Efficiency Using $W_{p}$ and Related Functions (Springer, 1991).

${ }^{17}$ H. Bauer, M. Beau, B. Friedl, C. Marchand, K. Miltner, and H. J. Reyher, Phys. Lett. A 146, 134 (1990). 